\title{
Fatores associados à automedicação em estudantes de enfermagem e enfermeiros: revisão integrativa de literatura
}

\author{
Factors associated with the self-medication by nursing students and nurses:integrative \\ literature review
}

\section{Factores asociados com la automedicación por estudiantes de enfermería y enfermeras: revisión integral de la literatura}

Tatiana Naiana Rodrigues dos Santos Porto ${ }^{1}$, Tatyanne Silva Rodrigues ${ }^{1}$, Luciana Stanford Baldoino $^{1 *}$, Ellen Maria de Sousa Santos ${ }^{1}$, Benedito Pereira de Sousa Neto ${ }^{1}$, Vinícius de Sousa Martins $^{1}$, Dorivaldo Pereira Carvalho ${ }^{1}$, Carla Patrícia Moreira Falcão ${ }^{1}$, Gaubeline Teixeira Feitosa ${ }^{1}$.

\section{RESUMO}

Objetivo: Identificar os fatores associados à prática da automedicação por estudantes de enfermagem e profissionais enfermeiros. Métodos: Revisão integrativa da literatura, realizada de agosto a novembro de 2019, na Scientific Eletronic Library Online (SciELO) e Literatura Latino-America e do Caribe em Ciências da Saúde (LILACS), Banco de Dados em Enfermagem (BDENF) via Biblioteca Virtual em Saúde (BVS). Resultados: Alguns fatores relacionados à automedicação mostram-se de risco para a escolha desta prática, sendo eles: o estresse laboral, sobrecarga de trabalho e falta de tempo para buscar consulta médica. Dos 9 artigos analisados na amostra final, o estresse correspondeu a $27 \%$, a sobrecarga e a falta de tempo representaram $18 \%$ respectivamente e o conhecimento sobre os medicamentos correspondeu a $37 \%$ dos fatores sociais evidenciados no estudo. Entre os fármacos mais consumidos estão os analgésicos (34\%), os antidepressivos (24\%), os antitérmicos (14\%), os anticoncepcionais (14\%) e os antibióticos (14\%). Considerações finais: Almeja-se que o estudo subsidie ações de promoção da saúde com esta população específica, assim como o desenvolvimento de outros estudos sobre o tema. A limitação principal do estudo foi a escassez de estudos originais voltados para o profissional de enfermagem e estudantes.

Palavras-chave: Autocuidado, Automedicação, Enfermagem, Estudantes de enfermagem.

\begin{abstract}
Objective: To identify the factors associated with the practice of self-medication by nursing students and professional nurses. Methods: Integrative literature review, conducted from August to November 2019, at the Scientific Electronic Library Online (SciELO) and Latin American and Caribbean Health Sciences Literature (LILACS), Database in Nursing (BDENF) via Virtual Health Library (VHL). Results: Some factors related to self-medication are at risk for choosing this practice, namely: work overload and lack of time to seek medical consultation. Of the 9 articles analyzed in the final sample, stress corresponded to $27 \%$, overload and lack of time accounted for $18 \%$ respectively, and knowledge about medications corresponded to $37 \%$ of the social factors highlighted in the study. Among the most consumed drugs are analgesics (34\%), antidepressants (24\%), antipyretics (14\%), contraceptives (14\%) and antibiotics (14\%). Final considerations: It is intended that the study subsidizes promotion actions with this specific population, as well as the development of other studies on the subject. The main limitation of the study was the scarcity of original studies aimed at nursing professionals and students.
\end{abstract}

Keywords: Self medication, Self care, Nursing, Nursing students.

\section{RESUMEN}

Objetivo: Identificar los factores asociados a la práctica de la automedicación por parte de estudiantes de enfermería y profesionales de la enfermería. Método: Revisión bibliográfica integradora, realizada de agosto a noviembre de 2019, en la Biblioteca Científica Electrónica en Línea (SciELO) y Literatura Latino Americana y del Caribe em Ciencias de la Salud (LILACS), Base de Datosen Enfermería (BDENF) por medio de la

${ }^{1}$ Faculdade de Ensino Superior Múltiplo (IESM), Timon - MA. *E- mail: Isbaldoino@hotmail.com

SUBMETIDO EM: $6 / 2020$

ACEITO EM: 7/2020

PUBLICADO EM: 10/2020 
Biblioteca Virtual en Salud (BVS). Resultados: Algunos factores relacionados con la automedicación están en riesgo para la elección de esta práctica, a saber: la sobrecarga de trabajo y la falta de tiempo para buscar consulta médica. De los 9 artículos analizados en la muestra final, el estrés correspondió al 27\%, la sobrecarga y la falta de tiempo el 18\% respectivamente, y el conocimiento sobre la medicación correspondió al $37 \%$ de los factores sociales destacados em el estudio. Entre los medicamentos más consumidos se encuentranlos analgésicos (34\%), los antidepresivos (24\%), los antipiréticos (14\%), los anticonceptivos (14\%) y los antibióticos (14\%). Consideraciones finales: Se pretende que el estudio subvencione acciones de promoción con esta población específica, así como el desarrollo de otros estudios sobre el tema. La principal limitación del estudiofue la escasez de estúdios originales dirigidos a profesionales y estudiantes de enfermería.

Palabras clave: Automedicación, Autocuidado, Enfermería, Estudiantes de enfermería.

\section{INTRODUÇÃO}

Automedicação é caracterizada como uma prática de consumo de medicamentos que ocorre sem a devida prescrição médica, ou seja, em que o praticante assume o risco de tal procedimento. Diversas vezes o indivíduo decide por esta conduta e faz o uso de medicamentos que considera ser o mais adequado para lidar com seus sintomas, levando em conta sua própria concepção (RIBEIRO LS, et al., 2018).

Diversas podem ser as razões que podem levar a prática da automedicação, dentre elas: a legislação brasileira que define os medicamentos de venda livre, a visão do medicamento como uma simples mercadoria, as práticas mercantilistas das indústrias farmacêuticas, a dificuldade de acesso da população a uma consulta médica, o grande número de farmácias e drogarias, a escassez de esclarecimento nas propagandas e publicidades sobre efeitos adversos que os medicamentos podem causar e outros (FERNANDES DL e MATOS LA, 2017).

O Brasil está entre os países que mais utilizam medicamentos no mundo. Possui cerca de 65 mil farmácias e drogarias, sendo três vezes maior do que o recomendado pela Organização Mundial da Saúde (OMS), que recomenda uma farmácia para 10 mil habitantes (ALVES DRF, et al., 2016). A prevalência de automedicação na população brasileira aumentou $16,1 \%$, de 2011 a 2015, se mostrando maior no sexo feminino, com faixa etária entre 20-39 anos, entre a raça indígena e parda, com nível de escolaridade igual ou superior a 12 anos de estudos, sendo maior na região Nordeste, já entre os profissionais mais adeptos desta prática estão os enfermeiros e os estudantes de enfermagem (ARRAIS PSD, et al., 2016).

Vale enfocar que o uso da autoterapia por estudantes e profissional de enfermagem, sem a prescrição médica de anti-inflamatórios, analgésicos e psico-fármacos, remete também aos riscos de reações alérgicas, intoxicações, e de interações medicamentosas. Com a propaganda intensa e a facilidade de acesso a medicamentos em farmácias e supermercados, dá a impressão de que estes são livres de riscos. Tais propagandas acabam estimulando o uso indiscriminado, que nem sempre resulta no efeito esperado pelo indivíduo, e expõem estes consumidores a reações indesejadas (SOUSA DRP e NETA ME, 2016).

A busca por intervenções terapêuticas sem a devida avaliação prévia por um profissional capacitado se dá também pela falta de recursos. Para os profissionais de enfermagem, as situações vivenciadas no dia a dia, longas jornadas de trabalho, remuneração inadequada, acumulação das escalas de serviço, desordens físicas e psíquicas e a exposição prolongada ao estresse podem causar comprometimentos significativos em sua saúde (RIBEIRO LS, et al., 2018).

Apesar da maioria dos medicamentos consumidos estarem isentos de prescrição médica, não se pode esquecer as possíveis intoxicações e efeitos adversos que eles podem vir a causar em seus usuários. No caso dos analgésicos e anti-inflamatórios não esteróides (AINES), pode-se citar os distúrbios gastrointestinais, as reações alérgicas e efeitos renais (ARRAIS PSD, et al., 2016).

É importante ressaltar que o consumo de medicamentos entre estudantes e os profissionais da área de saúde é uma conduta bastante comum e que pode aumentar ao longo do curso e na rotina de trabalho, estando este fato relacionado ao maior grau de informação sobre os agentes farmacológicos (ARAUJO CB, 2017).

Alguns fatores são favoráveis para o desencadeamento de doenças em profissionais de enfermagem: a sobrecarga de trabalho, dificuldade em delimitar papéis e funções entre esses profissionais, problemas nas 
relações interpessoais, além da falta de reconhecimento no seu trabalho. A potencialização de fatores como esses, resulta na maior possibilidade de falha no autocuidado (DOMINGUES JG, et al., 2019). No entanto, o estresse acomete o enfermeiro de forma tão avassaladora que o leva a utilizar psicofármacos, numa forma de aliviar as tensões diárias, o estresse é ser caracterizado como um desgaste do organismo, relacionado a situações onde o indivíduo é forçado ao limite, causando irritação e negação. Os enfermeiros são os profissionais que mais tem tendências a se tornarem dependentes das drogas psicoativas (VIEIRA GCG, et al., 2016).

Para obter a cura ou pelo menos o alívio de transtornos físicos e/ou psíquicos, vários profissionais de enfermagem recorrem ao uso de terapias medicamentosas, utilizando-se da prática da automedicaçãoo que figura uma conduta extremamente oposta ao conhecimento que estes possuem quanto ao uso irracional de medicamentos (CARSONI LMM e JUNIOR D, 2018). Neste sentido, os enfermeiros e os estudantes de enfermagem podem ser classificados como um grupo de risco, devido às continuas exposições e aos fatores que foram citados anteriormente.Desta forma, o uso de terapias farmacológicas acaba se tornando uma medida alternativa para alívio imediato dos sintomas (SOUSA DRP e NETA ME, 2016).

Seguindo a linha de pensamento do uso irracional de medicamentos, o estudante e o profissional que se submete ao uso de psicotrópicos, com frequência, apresenta outras experiências descontroladas, revelando assim mudanças de comportamento. Com a mudança do seu perfil passa a mostrar um desempenho indesejável: altos índices de absenteísmo, baixo rendimento, comportamento não colaborativo com a equipe, difícil convivência e desconforto no ambiente de trabalho, além de estar mais susceptível a erros e acidentes (LOPES AM e MATA LCC, 2016).

O tema relacionado aos profissionais de saúde e a automedicação é pouco abordado na produção científica, pois existe um paradoxo entre o papel do profissional de saúde que de cuidador, torna-se o paciente, o que causa dificuldade de aceitação do profissional como agente que necessita de cuidados, o que colabora para que o problema se agrave. E por fim, esta pesquisa justifica-se pelo fato da vulnerabilidade dos enfermeiros em relação ao consumo de medicamentos ser um grave problema de saúde pública e ainda tão pouco estudado. Diante do exposto, o presente estudo tem como objetivo identificar os fatores associados à prática da automedicação por estudantes de enfermagem e profissionais enfermeiros.

\section{MÉTODOS}

Trata-se de uma revisão integrativa da literatura. A revisão integrativa de literatura baseia-se em etapas distintas que se unem para o alcance do objetivo final, sendo elas: a identificação de tema e onde se define a questão norteadora do estudo, a definição dos critérios de inclusão e de exclusão, a identificação dos estudos pré-selecionados, a categorização dos estudos selecionados para amostra final, análise e interpretação dos resultados e apresentação da síntese do conhecimento (MENDES KDS, et al., 2019).

Uma ótima estratégia para ajudar a construir a questão da pesquisa é uma estratégia chamada PICO. Uma questão de pesquisa bem construída possibilita a definição adequada das informações (evidências) necessárias para a resposta do aspecto clínico da pesquisa, maximizando a recuperação de evidências nas bases de dados e bibliotecas virtuais e desta forma evitar buscas desnecessárias (SANTOS CMC, et al., 2007).

A questão de pesquisa foi organizada de acordo com a estratégia PICO (P: População; I: Intervenção; C: Comparação; O: Desfecho). Portanto, foi sintetizada a seguinte estrutura: $\mathrm{P}$ - Estudantes de Enfermagem e Enfermeiros; I - Automedicação; C - Sem comparação; O - Fatores de risco. Por tanto, a questão norteadora é: Quais os fatores associados à prática da automedicação por estudantes de enfermagem e profissionais enfermeiros disponíveis na literatura?

Para a realização do levantamento bibliográfico, os descritores utilizados encontram-se presentes no Banco de Descritores em Ciências da Saúde (DeCS) e baseado no objetivo da pesquisa, optou-se por: "Autocuidado, Automedicação", "Enfermagem" e "Estudantes de Enfermagem".

Como forma de refinar a coleta da amostragem, utilizou os operadores booleanos "AND", para conectar os descritores, e "OR" para conectar as palavras-chave. Foi utilizada a biblioteca virtual da Scientific Eletronic Library Online (SciELO), a base de dados Literatura Latino-America e do Caribe em Ciências da Saúde 
(LILACS) e Banco de Dados em Enfermagem (BDENF) via Biblioteca Virtual em Saúde (BVS). A coleta de dados ocorreu de agosto à novembro de 2019.

Os critérios de inclusão foram artigos originais, publicados entre2015 a 2019, disponíveis em inglês, português e espanhol que discorriam sobre a automedicação em estudantes e profissionais de enfermagem. Já como critério de exclusão optou-se por não utilizar textos incompletos, teses, resumos, cartas ao editor, artigos que não estivessem disponíveis na íntegra on-line e que relacionassem a automedicação à outra classe profissional (Figura 1).

Figura 1- Fluxograma da aplicação das etapas dos critérios de inclusão e exclusão dos artigos para amostra final.

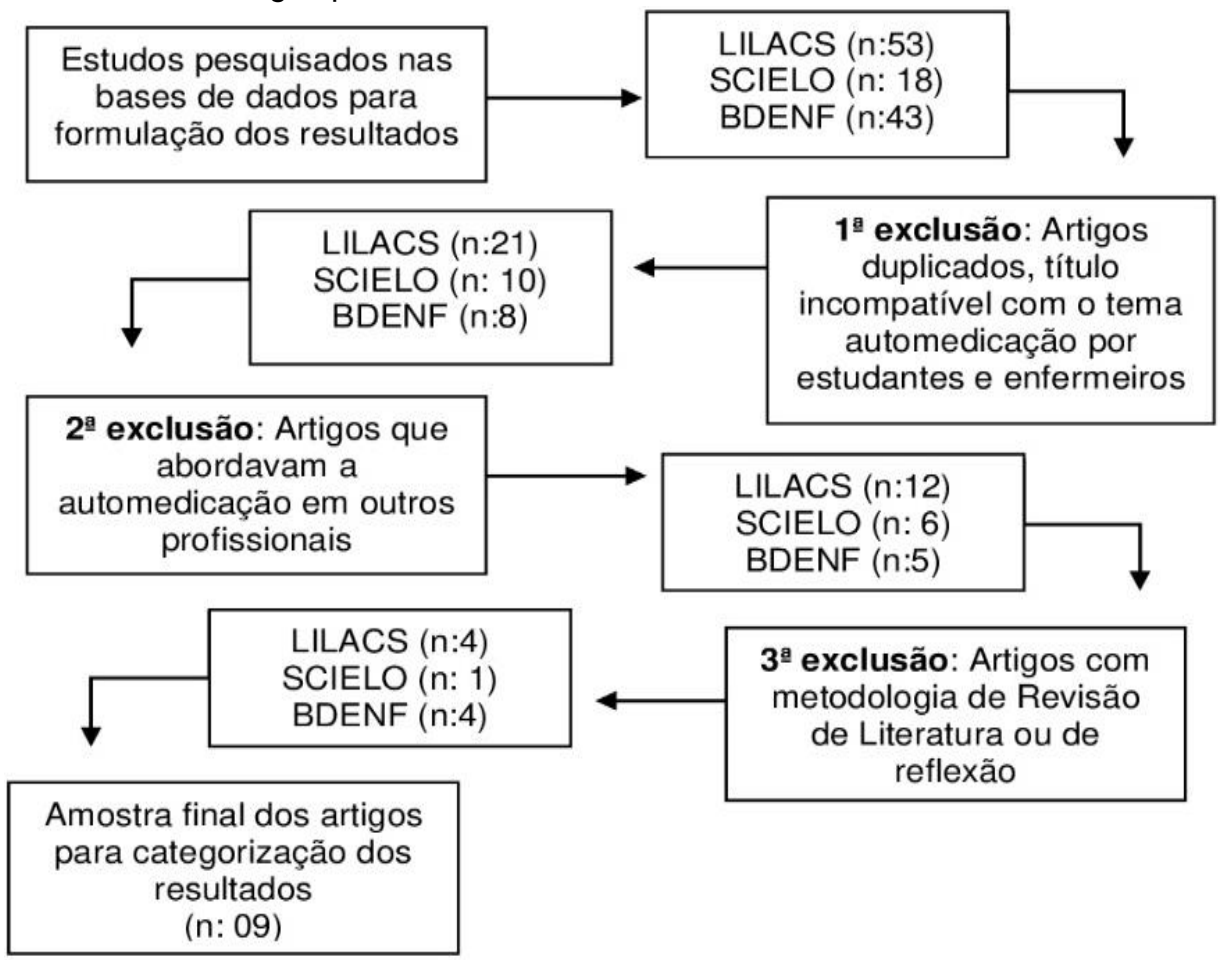

Fonte: Porto TNRS, et al., 2020.

Realizou-se então uma leitura do título e do resumo dos artigos, onde foram excluídos aqueles que não abordavam questão do estudo, artigos duplicados também foram excluídos, além dos que não forneciam acesso completo ao texto. Após esta primeira seleção, realizou- se uma leitura completa dos artigos. Desta forma, compôs a amostra final nove artigos.

Após estas etapas, realizou- se a avaliação das evidências por meio da leitura criteriosa dos artigos e fichamento por meio de um instrumento construído anteriormente, com informações importantes, tais como: Autor (es), Periódico/Ano de publicação, Tipo de estudo/Metodologia, População, Fatores relacionados a automedicação, Medicamentos utilizados e Qualis capes.

\section{RESULTADOS E DISCUSSÃO}

Os dados encontrados foram apresentados em quadro esquemático, refletindo a síntese das características dos estudos incluídos e outra parte foi apresentada de forma descritiva para um melhor entendimento do conteúdo dos artigos. Posteriormente seguiu-se a análise e a discussão dos resultados fundamentada no diálogo dos autores que discutem o tema (Quadro 1). 
Quadro 1 - Caracterização dos estudos segundo: Autores, Periódico /Ano de publicação, Tipo de estudo/Metodologia, População do estudo, Fatores relacionados a automedicação, Medicamentos utilizados, qualis capes e nível de evidência.

\begin{tabular}{|c|c|c|c|c|c|c|c|}
\hline Autor (es) & Título do artigo & $\begin{array}{l}\text { Períodico/ano } \\
\text { de publicação }\end{array}$ & $\begin{array}{c}\text { Tipo de } \\
\text { estudo/metodologia }\end{array}$ & $\begin{array}{l}\text { População } \\
\text { do estudo }\end{array}$ & $\begin{array}{l}\text { Fatores relacionados a } \\
\text { automedicação }\end{array}$ & $\begin{array}{l}\text { Medicamentos } \\
\text { utilizados }\end{array}$ & $\begin{array}{l}\text { Qualis } \\
\text { Capes }\end{array}$ \\
\hline $\begin{array}{l}\text { VIEIRA GCG, et } \\
\text { al. }\end{array}$ & $\begin{array}{l}\text { Uso de psicotrópicos pelo } \\
\text { enfermeiro: sua relação } \\
\text { com o trabalho }\end{array}$ & $\begin{array}{c}\text { Revista } \\
\text { Cinergis/ } 2016\end{array}$ & $\begin{array}{l}\text { Pesquisa qualitativa do } \\
\text { tipo descritivo- } \\
\text { exploratória }\end{array}$ & $\begin{array}{c}17 \\
\text { Enfermeiros } \\
\text { (as) }\end{array}$ & $\begin{array}{l}\text { Sobrecarga de trabalho, } \\
\text { relações pessoais, } \\
\text { desgaste físico e psíquico. }\end{array}$ & $\begin{array}{l}\text { Antidepressivos, } \\
\text { Ansiolíticos e } \\
\text { Analgésicos }\end{array}$ & B3 \\
\hline $\begin{array}{l}\text { RODRIGUES } \\
\text { WP, et al. }\end{array}$ & $\begin{array}{l}\text { La automedicación entre } \\
\text { los profesionales de la } \\
\text { saluden Fátima/Bahia }\end{array}$ & $\begin{array}{l}\text { Revista } \\
\text { Eletrônica } \\
\text { Acervo } \\
\text { Saúde/2019 }\end{array}$ & $\begin{array}{l}\text { Estudo descritivo, } \\
\text { caráter exploratório e } \\
\text { corte transversal. }\end{array}$ & $\begin{array}{l}45 \\
\text { Enfermeiros } \\
\quad \text { (as) }\end{array}$ & $\begin{array}{c}\text { Estresse laboral, dor e } \\
\text { desgaste físico e psíquico }\end{array}$ & $\begin{array}{l}\text { Dipirona, Paracetamol, } \\
\text { Ibuprofeno, Prednisona, } \\
\text { Diazepan e Dorflex. }\end{array}$ & B2 \\
\hline ALI AS, et al. & $\begin{array}{c}\text { Practices of self- } \\
\text { medication with antibiotics } \\
\text { among nursing students of } \\
\text { Institute of Nursing, Dow } \\
\text { University of Health } \\
\text { Sciences, Karachi, } \\
\text { Pakistan }\end{array}$ & $\begin{array}{l}\text { Journal Pakistan } \\
\text { Med Assoc/2016 }\end{array}$ & Estudo Transversal & $\begin{array}{c}160 \\
\text { Estudantes } \\
\text { de } \\
\text { Enfermagem }\end{array}$ & $\begin{array}{l}\text { Conhecimento sobre } \\
\text { medicamentos, } \\
\text { conveniência. }\end{array}$ & Antibióticos & B2 \\
\hline $\begin{array}{l}\text { ALVES DRF, et } \\
\text { al. }\end{array}$ & $\begin{array}{l}\text { Self-medication: practice } \\
\text { among nursing } \\
\text { undergraduates }\end{array}$ & $\begin{array}{c}\text { Journal Nursing } \\
\text { UFPE } \\
\text { online/2019 }\end{array}$ & $\begin{array}{l}\text { Estudo quantitativo, } \\
\text { exploratório e descritivo }\end{array}$ & $\begin{array}{c}100 \\
\text { Estudantes } \\
\text { de } \\
\text { Enfermagem }\end{array}$ & $\begin{array}{c}\text { Falta de acessibilidade a } \\
\text { serviços de saúde, } \\
\text { limitação de recursos } \\
\text { financeiros, falta de } \\
\text { tempo. }\end{array}$ & $\begin{array}{l}\text { Antitérmicos, } \\
\text { Analgésicos, Xaropes } \\
\text { para tosse, } \\
\text { Antiasmáticos e } \\
\text { Antiácidos. }\end{array}$ & B4 \\
\hline $\begin{array}{l}\text { GAMA ASM, } \\
\text { SECOLI SR. }\end{array}$ & $\begin{array}{c}\text { Automedicação em } \\
\text { estudantes de enfermagem } \\
\text { do Estado do Amazonas - } \\
\text { Brasil }\end{array}$ & $\begin{array}{l}\text { Revista Gaúcha } \\
\text { de Enfermagem/ } \\
2017\end{array}$ & Estudo Transversal & $\begin{array}{c}116 \\
\text { Estudantes } \\
\text { de } \\
\text { Enfermagem }\end{array}$ & $\begin{array}{l}\text { Uso de prescrições a } \\
\text { anteriores, conhecimento } \\
\text { sobre medicamentos, } \\
\text { indicação de familiares e } \\
\text { propagandas em mídia. }\end{array}$ & $\begin{array}{l}\text { Anti-inflamatórios, Anti- } \\
\text { histamínicos, } \\
\text { Antimicrobianos, } \\
\text { Anticoncepcionais, } \\
\text { Analgésicos e } \\
\text { Antitérmicos. }\end{array}$ & B1 \\
\hline
\end{tabular}

REAS/EJCH | Vol.12(10) | e4111 | DOI: https://doi.org/10.25248/reas.e4111.2020 Página 5 de 11 


\section{Revista Eletrônica Acervo Saúde / Electronic Journal Collection Health | ISSN 2178-2091}

\begin{tabular}{|c|c|c|c|c|c|c|c|}
\hline Autor (es) & Título do artigo & $\begin{array}{l}\text { Períodico/ano } \\
\text { de publicação }\end{array}$ & $\begin{array}{c}\text { Tipo de } \\
\text { estudo/metodologia }\end{array}$ & $\begin{array}{l}\text { População } \\
\text { do estudo }\end{array}$ & $\begin{array}{l}\text { Fatores relacionados a } \\
\text { automedicação }\end{array}$ & $\begin{array}{l}\text { Medicamentos } \\
\text { utilizados }\end{array}$ & $\begin{array}{l}\text { Qualis } \\
\text { Capes }\end{array}$ \\
\hline SADO E, et al. & $\begin{array}{c}\text { Epidemiology of } \\
\text { self-medication with } \\
\text { modern medicines among } \\
\text { health care professionals in } \\
\text { Nekemte town, western } \\
\text { Ethiopia }\end{array}$ & $\begin{array}{l}\text { BMC Research } \\
\text { Notes/ } 2017\end{array}$ & Estudo Transversal & $\begin{array}{l}88 \\
\text { Enfermeiros } \\
\quad \text { (as) }\end{array}$ & $\begin{array}{l}\text { Limitação de recursos } \\
\text { financeiros, conhecimento } \\
\text { sobre medicamentos. }\end{array}$ & $\begin{array}{l}\text { Analgésicos, Antibióticos, } \\
\text { Contraceptivos orais e } \\
\text { Antiácidos. }\end{array}$ & B3 \\
\hline $\begin{array}{l}\text { ROCHA PR, } \\
\text { DAVID HMSL. }\end{array}$ & $\begin{array}{l}\text { Padrão de consumo de } \\
\text { álcool e outras drogas } \\
\text { entre profissionais de } \\
\text { saúde: retrato de alunos de } \\
\text { cursos lato sensu de uma } \\
\text { instituição pública }\end{array}$ & $\begin{array}{c}\text { Revista } \\
\text { Eletrônica } \\
\text { Saúde Mental } \\
\text { Álcool Drogas/ } \\
2015\end{array}$ & $\begin{array}{l}\text { Estudo quantitativo, } \\
\text { transversal, descritivo, } \\
\text { exploratório. }\end{array}$ & $\begin{array}{l}120 \\
\text { Enfermeiros } \\
\quad(\text { as })\end{array}$ & $\begin{array}{l}\text { Tensão laboral, } \\
\text { Ansiedade, carga de } \\
\text { trabalho elevada e má } \\
\text { remuneração. }\end{array}$ & Ansiolíticos e Sedativos. & B2 \\
\hline $\begin{array}{l}\text { HOFFMANN } \\
\text { AMM, et al. }\end{array}$ & $\begin{array}{l}\text { Automedicação entre } \\
\text { acadêmicos de } \\
\text { Enfermagem }\end{array}$ & $\begin{array}{l}\text { Revista } \\
\text { Eletrônica } \\
\text { Acervo } \\
\text { Saúde/2017 }\end{array}$ & $\begin{array}{l}\text { Estudo de campo } \\
\text { transversal e } \\
\text { delineamento } \\
\text { quantitativo }\end{array}$ & $\begin{array}{c}426 \\
\text { Estudantes } \\
\quad \text { de } \\
\text { Enfermagem }\end{array}$ & $\begin{array}{c}\text { Fácil acesso aos } \\
\text { medicamentos, falta de } \\
\text { tempo e segurança na } \\
\text { utilização de } \\
\text { medicamentos. }\end{array}$ & $\begin{array}{l}\text { Analgésicos, } \\
\text { Anticoncepcionais, } \\
\text { Antibióticos e } \\
\text { Antipiréticos. }\end{array}$ & B2 \\
\hline $\begin{array}{l}\text { SCHNEIDER } \\
\text { APH, AZAMBUJA } \\
\text { PG. }\end{array}$ & $\begin{array}{c}\text { Uso de fármacos } \\
\text { psicotrópicos por } \\
\text { profissionais da saúde } \\
\text { atuantes da área hospitalar }\end{array}$ & $\begin{array}{l}\text { Revista Infarma } \\
\text { Ciências } \\
\text { Farmacêuticas/ } \\
2015\end{array}$ & $\begin{array}{c}\text { Estudo analítico } \\
\text { observacional } \\
\text { transversal com } \\
\text { abordagem quantitativa }\end{array}$ & $\begin{array}{c}106 \\
\text { Profissionais } \\
\text { de } \\
\text { Enfermagem }\end{array}$ & $\begin{array}{l}\text { Depressão, ansiedade e } \\
\text { insônia. }\end{array}$ & $\begin{array}{l}\text { Ansiolíticos, sedativos e } \\
\text { Analgésicos }\end{array}$ & B3 \\
\hline
\end{tabular}

Fonte: Porto TNRS, et al., 2020 
A prática da automedicação vem aumentando sobremaneira, ela se mostra crescente principalmente entre profissionais da saúde, em especial enfermeiros que mesmo antes do término da graduação sentem- se seguros na utilização de medicamentos sem prescrição médica, levando em conta apenas o conhecimento que adquirem durante $o$ curso.

Alguns fatores relacionados a automedicação mostram-se de risco para a escolha desta prática entre estudantes e profissionais enfermeiros, segundo os estudos analisados, o estresse laboral, sobrecarga de trabalho e falta de tempo para buscar consulta médica foram apontados. Os conhecimentos sobre os medicamentos correspondem a $37 \%$ dos fatores sociais evidenciados no estudo (Gráfico 1).

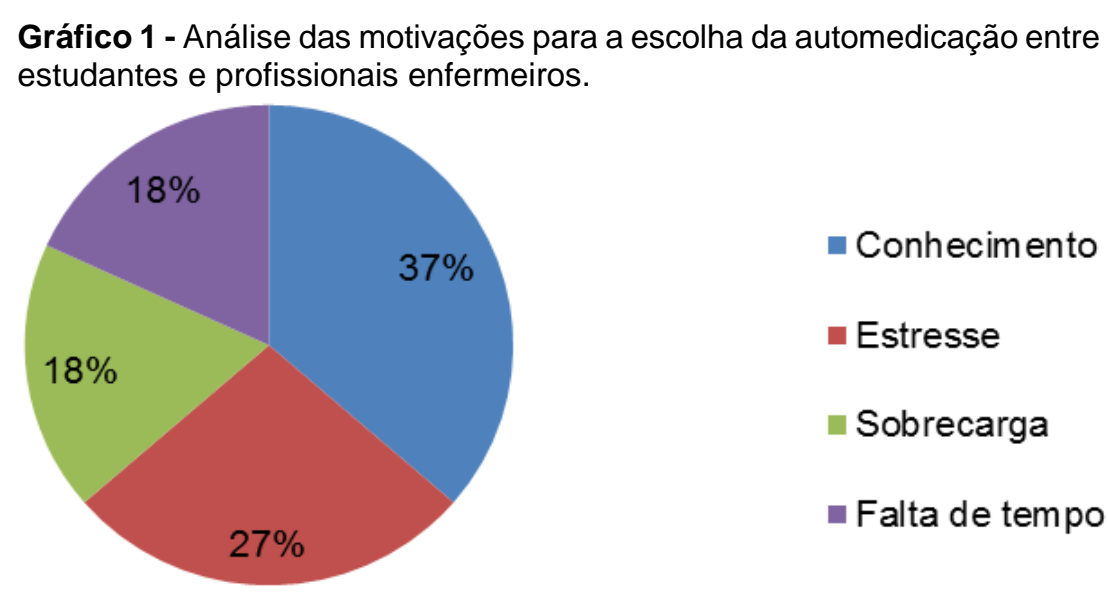

Fonte: Porto TNRS, et al., 2020.

A automedicação muitas vezes acontece porque as pessoas obtêm bons resultados e continuam consumindo, o que acarreta danos à saúde, e o uso indiscriminado desses fármacos pode trazer consequências para o indivíduo quando não há acompanhamento médico ou até uma assessoria farmacêutica, sendo esta uma prática que pode resultar uma enfermidade ou até mesmo prejudicar um tratamento adequado.

A dor de cabeça foi citada com frequência nos estudos selecionados nesta pesquisa, algo que pode estar relacionado ao estresse laboral destes profissionais e estudantes, visto a quantidade de responsabilidades acadêmicas e profissionais e também por lidarem constantemente com o sofrimento humano. Seja para tratar algum sintoma ou apenas para alívio, a facilidade da obtenção destes medicamentos torna- se um facilitador para a automedicação, visto que a maioria destes medicamentos pode ser conseguida sem a necessidade de receituário médico. Entre os fármacos mais consumidos estão os analgésicos (34\%), os antidepressivos (24\%), os antitérmicos (14\%), os anticoncepcionais (14\%) e os antibióticos (14\%) (Gráfico 2).

Gráfico 2 - Principais medicamentos utilizados por estudantes de enfermagem e profissionais enfermeiros.

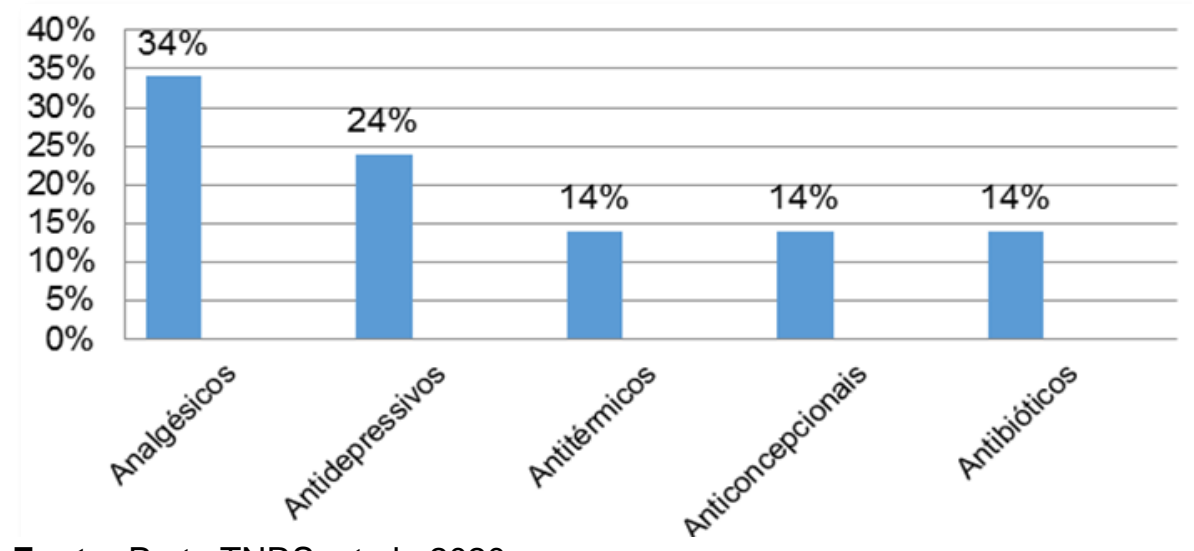

Fonte: Porto TNRS, et al., 2020. 
Trabalhadores da saúde estão frequentemente envolvidos em atividades complexas, o que ocasiona desgaste psicoemocional comprometendo a saúde dos mesmos e a qualidade do serviço prestado. Os principais problemas de saúde relatados pelos trabalhadores referem-se aos sistemas musculoesquelético, respiratório e nervoso. Essas queixas equivalem a 73,4\% de todas as referidas pelos trabalhadores de saúde, o que explica o grande consumo de analgésicos e antidepressivos entre estes profissionais (PEREIRA IVS, et al., 2014).

Pesquisa realizada por Arrais PSD, et al.(2016) mostra que a população feminina mais susceptível à prática da automedicação, porém de o grau de escolaridade não parecer ser um fator diferencial, visto que o uso irracional de medicamentos foi detectado em todas as classes sociais com níveis de escolaridade distintas, a pesquisa demonstrou ainda, uma prevalência do uso irracional de medicamentos em todas as regiões do Brasil, com crescimento superior na região Nordeste e Centro-oeste, mostrando assim uma prática bastante comum na população brasileira.

Em estudo realizado no Paraná, cerca de $70 \%$ dos enfermeiros afirmaram fazer uso de algum medicamento, sendo apenas $30 \%$ de uso contínuo com acompanhamento médico e $44 \%$ de maneira descontínua e de escolha própria. Os resultados mostraram que a busca e utilização pela medicação tem relação com a sobrecarga de trabalho, ansiedade, cansaço e estresse.

Desta forma, muitos passam a fazer uso de substâncias psicoativas, mesmo que sem orientação médica, em busca de alívio da sobrecarga de trabalho físico e emocional que leva ao comprometimento da qualidade de vida do profissional e das atividades realizadas (VIEIRA GCG, et al., 2016).

Dentre os profissionais que fazem uso regular de medicamentos psicoativos, as justificativas mencionadas para a escolha foram: relaxar quando está tenso (12\%), celebrar situações especiais (12\%), tirar ansiedade (10\%) e alegrar-se quando está triste (8\%) (ROCHA PR e DAVID HMSL, 2015).

Os fatores que encorajam a prática da automedicação estão relacionados ao estresse laboral, ao medo de adquirirem algum problema de saúde e/ou comportamento de risco à saúde como mostram estatísticas que reforçam tais fatores, cerca de $30 \%$ dos enfermeiros estão mais susceptíveis que a população em geral a se tornarem dependentes de medicamentos e a apresentarem alguma síndrome relacionada à exaustão laboral, se em comparação com outros profissionais (SANTOS ANM, et al., 2018).

Para Rodrigues WP, et al. (2019) em sua pesquisa observaram que $64,4 \%$ dos enfermeiros admitiram ter utilizado algum medicamento nos últimos 10 dias. Quando questionado se o medicamento utilizado foi por prescrição médica, a maioria dos profissionais $(44,4 \%)$ respondeu de forma afirmativa, seguido dos profissionais $(35 \%)$ que não responderam à pergunta e somente $(20,0 \%)$ responderam que não.

Com base nos dados coletados, os principais medicamentos usados pelos profissionais foram: Dipirona (31,1\%), Paracetamol (24,4\%), Ibuprofeno (20\%), Prednisona (11,1\%), Diazepam (6,7\%) e Dorflex (Citrato de Orfenadrina + Dipirona Sódica + Cafeína) (6,7\%). Em pesquisa realizada no Amazonas, dentre os medicamentos utilizados através da automedicação por estudantes de enfermagem, os mais consumidos foram paracetamol e dipirona (48,8\%), cefalexina $(6,0 \%)$ e complexo B $(8,3 \%)$. Já entre os antimicrobianos, os mais utilizados foram a cefalexina $(55,6 \%)$, amoxicilina $(22,2 \%)$, ampicilina $(11,1 \%)$ e azitromicina $(11,1 \%)$. A automedicação, segundo os estudantes, foi influenciada por familiares e amigos (36,4\%), utilização de prescrições anteriores $(30,7 \%)$, conhecimento sobre medicamentos $(15,9 \%)$ e propagandas em mídias (12,5\%) (GAMA ASM e SECOLI SR, 2017).

As classes dos medicamentos mais utilizados pelos estudantes de enfermagem, segundo a pesquisa de Hoffmann AMM, et al. (2017) foram: analgésicos (41,03\%) e anticoncepcionais (15,30\%), seguido dos antibióticos (12,53\%) e antipiréticos (8,84\%). A maioria dos universitários $(75,89 \%)$ disseram comprar os medicamentos em farmácia sem receita médica $(8,45 \%)$ ou utilizam medicamentos que sobram de pacientes e que não foram devolvidos à farmácia no trabalho. $O$ fácil acesso aos medicamentos foi o motivo que os levaram a praticar a automedicação sendo $(49,80 \%)$ dos estudantes e $(26,41 \%)$ citaram a falta de tempo. 
Em relação à equipe de enfermagem a prática desde o período acadêmica é comum e se torna maior depois de formado. Essa situação é preocupante principalmente por serem profissionais que estão ligados com as atividades de promoção e de prevenção da saúde, bem como na prescrição e administração dos medicamentos, se tornando o maior percentual dentro dos hospitais e serviços de saúde, e o primeiro profissional na linha de cuidado aos pacientes (MATOS JF, et al., 2018).

De forma geral, a automedicação e os fatores associados estão envolvidos em uma variedade de eventos que pode mudar de acordo com a população e também com a cultura. As ofertas precárias dos serviços de saúde no Brasil desempenham um papel importante na automedicação, estando associado à facilidade de aquisição dos medicamentos sem prescrição médica e sem orientação farmacêutica, além do elevado número de farmácias, assim como dificuldade de consulta médica, fortalece essa prática (MORTAZAVI SS, et al., 2017).

A equipe de enfermagem é responsável por prestar o cuidado seguro e de qualidade, com foco no paciente e na coletividade, seu cuidado é estabelecido através de orientações, decisões precisas e discutidas pela equipe multiprofissional, melhorando a promoção de saúde e diminuindo o risco de outras doenças (OLIVEIRA JKA, et al., 2018).

No estudo realizado na Etiópia, com o objetivo de descrever quais os medicamentos eram utilizados na prática da automedicação, dos 154 entrevistados, (67\%) deles afirmaram ter utilizados medicamentos modernos nos últimos 2 meses sem prescrição médica, os medicamentos mais utilizados foram: analgésicos (36,1\%) e antibióticos (23,9\%). Já a familiaridade com os medicamentos (24\%) foi o motivo mais citado, seguido por estado civil (SADO E, et al., 2017).

Os antibióticos são substâncias químicas, que podem ser naturais ou sintéticas, usados com finalidade de prevenir ou tratar infecções causadas por microrganismos patógenos. Porém seu uso indiscriminado e indevido, muitas vezes sem prescrição, sem dose e sem indicação, ocasiona uma exacerbada resistência microbiana, causando assim a ineficiência do medicamento (OLIVEIRA HJP, et al., 2017).

Em seu estudo Ali AS, et al. (2016) realizado no Paquistão, a maioria dos participantes $(60,8 \%)$ relataram ter completa satisfação com a automedicação com antibióticos, $44(55,7 \%)$ citaram a automedicação com antibióticos como uma prática aceitável, enquanto que apenas $23(29,1 \%)$ disseram não aceitar a escolha de tal prática por um profissional da saúde.

Na grande maioria $(52,7 \%)$ assumiram já ter se automedicado com antibióticos sem prescrição. Dos motivos que os levaram a se automedicarem, os mais citados foram o conhecimento sobre o medicamento $(74,7 \%)$ e a conveniência $(16,5 \%)$. Febre $(46,8 \%)$ e a dor de garganta $(34,2 \%)$ foram os sintomas mais comuns que os influenciaram à automedicação.

No que diz respeito aos estudantes da área da enfermagem, estudos conduzidos por Leite ICPCR, et al. (2016) mostram prevalência da automedicação entre $88,57 \%$ neste grupo, equivalente aos resultados da pesquisa de Gama ASM e Secoli SR (2017) (76\%) que relatam que 46,6\% dos estudantes participantes de sua pesquisa referem não acreditar na necessidade de avaliação médica e Lopes AM e Mata LCC (2016) (54\%) que em que $38 \%$ dos participantes de sua pesquisa referiram tal hábito (RIBEIRO LS, et al., 2018).

A automedicação é potencialmente prejudicial à saúde do profissional de enfermagem e também da coletividade, porque mesmo os medicamentos sem restrição, considerados simples e sem reações adversas, podem levar a uma reação de hipersensibilidade, hemorragias digestivas, além de mascarar sintomas de doenças mais graves (IURAS A, et al., 2016).

Alves DRF, et al. (2019) em seu estudo mostrou que, quando os estudantes de enfermagem e enfermeiros são questionados sobre por quem são orientados a realizar a automedicação, a orientação própria é a principal responsável pela prática com $(71,0 \%)$, de familiares responde por $(62,0 \%)$, funcionários de farmácias $(25,0 \%)$, os amigos $(20,0 \%)$, informações vistas a internet representam $(15,0 \%)$ e $(8,0 \%)$ disseram ter outras fontes de orientações. Os dados revelaram que quase totalidade dos investigados (99,0\%) afirmou praticar a automedicação, enquanto que apenas $(1,0 \%)$ referiu nunca ter feito uso de medicamentos sem a prescrição médica. 
Em relação à área de trabalho, o maior consumo foi entre os profissionais atuantes em Unidades de Terapia Intensiva $(34,78 \%)$ e Unidades de Internação $(34,78 \%)$, com a carga horária semanal de trabalho superior a 60 horas $(50 \%)$, incluindo também as situações onde o profissional tem mais de $\mathrm{m}$ vínculo empregatício.

Os principais motivos para o uso dos medicamentos foram a depressão, a ansiedade e a insônia. A falta de conhecimento sobre as características dos medicamentos e tratamento também foram citadas (SCHNEIDER APH e AZAMBUJA PG, 2015).

\section{CONSIDERAÇÕES FINAIS}

Evidenciou-se o risco da automedicação por estudantes e profissionais enfermeiros, sendo que o conhecimento sobre os medicamentos corresponde a $37 \%$ dos fatores sociais onde constatou no estudo, o estresse foi citado em $27 \%$ dos casos além da sobrecarga e a falta de tempo para ir às consultas médicas (18\%). Almeja-se que esta pesquisa subsidie ações de promoção da saúde com esta população específica, como também o desenvolvimento de outros estudos sobre o tema além da limitação devidoà escassez de estudos originais voltados a este público. Pois, percebe-se que os mesmos possuem conhecimento sobre as drogas disponíveis e seus efeitos. E por fim, a automedicação sem o acompanhamento médico pode-se tornar abusivo e/ou indiscriminado e do risco de efeitos indesejáveis.

\section{REFERÊNCIAS}

1. ALI AS, et al. Practices of self-medication with antibiotics among nursing students of Institute of Nursing, Dow University of Health Sciences, Karachi, Pakistan. Journal Pakistan Med Assoc, Pakistan, 2016; 66(2): 235-237.

2. ALVES DRF, et al. Self-medication: practice among nursing undergraduates. Journal Nursing UFPE online, Recife, 2019; 13(2): 363-70.

3. ARRAIS PSD, et al. Prevalência da automedicação no Brasil e fatores associados. Revista de Saúde Pública, São Paulo, 2016; 50(2): 1-13.

4. ARAÚJO CB. Automedicação entre os profissionais da saúde. 2017. 32 páginas. Trabalho de Conclusão de Curso (Graduação em Enfermagem) - Centro Universitário Anhanguera, Campo Grande, 2017.

5. CARSONI LMM, JUNIOR D. A. Marketing farmacêutico: relação das publicidades televisivas com a automedicação. Revista Visão Acadêmica, Curitiba, 2018; 19(4): 55-66.

6. DOMINGUES JG, et al. Doenças crônicas não transmissíveis em profissionais de enfermagem de um hospital filantrópico no Sul do Brasil. Epidemiologia e Serviços de Saúde, Brasília, 2019; 28(2).

7. FERNANDES DL, MATOS LA. A Relação da Propaganda de Medicamento e a Automedicação. 2 ed. São Paulo-SP, 2017. 25p.

8. GAMA ASM, SECOLI SR. Automedicação em estudantes de enfermagem do Estado do Amazonas - Brasil. Revista Gaúcha de Enfermagem, Porto Alegre, 2017; 38(1).

9. HOFFMANN AMM, et al. Automedicação entre acadêmicos de Enfermagem. Revista Eletrônica Acervo Saúde, Belo Horizonte- MG, 2017; supl(9): 842-848.

10. IURAS A, et al. Prevalência da automedicação entre estudantes da Universidade do Estado do Amazonas (Brasil), Revista Portuguesa de Estomatologia, Medicina Dentária e Cirurgia Maxilofacial. 2016; 57(2): 104-111.

11. LEITE ICPCR, et al. Automedicação em acadêmicos: um estudo transversal, Programa de Pós-Graduação em Ciências Farmacêuticas, Universidade Federal do Piauí, Boletim Informativo Geum, 2016; 7(1): $19-27$.

12. LOPES AM, MATA LCC. Automedicação entre graduandos das áreas de saúde e exatas da faculdade ciências da vida na cidade de sete lagoas/MG. Faculdade Ciências da Vida, Sete Lagoas - MG, 2016.

13. MATOS JF, et al. Prevalência, perfil e fatores associados à automedicação em adolescentes e servidores de uma escola pública profissionalizante. Cad. Saúde Coletiva, Rio de Janeiro, 2018; 26(1): 76-83.

14. MENDES KDS, et al. Uso de gerenciador de referências bibliográficas na seleção dos estudos primários em revisão integrativa. Texto contexto- enfermagem. Curitiba, 2019; 28(epud 14).

15. MORTAZAVI SS, et al. Self-medication among the elderly in Iran: a content analysis study. BMC geriatrics, 2017; 17(1): 198.

16. OLIVEIRA HJP, et al. Educação em saúde como forma preventiva do uso indiscriminado dos antibióticos. Revista Saúde, Recife- PE, 2017; 11(1): 52- 58.

17. OLIVEIRA JKA, et al. Patient safety in nursing care during medication administration. Revista Latino-Am. Enfermagem, 2018; 26(9): 3-17.

18. PEREIRA IVS, et al. Morbidade autorreferida por trabalhadores das Equipes de Saúde da Família. Ciência \& Saúde Coletiva, Minas Gerais, 2014; 19(2): 461-468.

19. RIBEIRO LS, et al. Automedicação entre estudantes e profissionais de enfermagem. Revista Enfermagem em Evidência, São Paulo, 2018; 2(1): 15-27. 
20. RODRIGUES WP, et al. La automedicación entre los profesionales de la saluden Fátima/Bahia. Revista Eletrônica Acervo Saúde, Bahia, 2019; 11(12).

21. ROCHA PR, DAVID HMSL. Padrão de consumo de álcool e outras drogas entre profissionais de saúde: retrato de alunos de cursos lato sensu de uma instituição pública. Revista Eletrônica Saúde Mental Álcool Drogas, Rio de Janeiro, 2015; 11(1): 41-48.

22. SADO E, et al. Epidemiology of self-medication with modern medicines among health care professionals in Nekemte town, western Ethiopia. BMC Res Notes, Nekemte, 2017; 10(4): 533.

23. SANTOS CMC, et al. A estratégia pico para a construção da pergunta de pesquisa e busca de evidências. Revista Latino- americana de Enfermagem, 2007; 15(3).

24. SANTOS ANM, et al. Self-medication among participants of an Open University of the Third Age and associated factors. Revista bras. geriatr. gerontol. Rio de Janeiro, 2018; 21(4): 419-427.

25. SOUZA DRP, NETA ME. Automedicação por profissionais e acadêmicos da área da saúde: uma revisão de literatura. Revista da Universidade Vale do Rio Verde, Três corações-MG, 2016; 14(2): 965-974.

26. SCHNEIDER APH, AZAMBUJA PG. Uso de fármacos psicotrópicos por profissionais da saúde atuantes da área hospitalar. Revista Infarma-Ciências Farmacêuticas, Brasília, 2015; 27(1): 14-21.

27. VIEIRA GCG, et al. Uso de psicotrópicos pelo enfermeiro: sua relação com o trabalho. Revista Cinergis, Santa Cruz do Sul, 2016; 17(3): 191-195. 